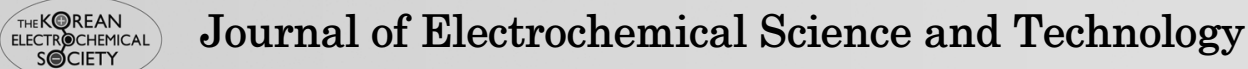
jecst.org

\title{
Biomimetic Copper Complex Containing Polymer Modified Electrode for Electrocatalytic Reduction of Oxygen
}

\author{
D. Saravanakumar ${ }^{1,2 *}$, Rajaram Krishna Nagarale ${ }^{1}$, Harish Chandra Jirimali ${ }^{1}$, Jong Myung Lee $^{1}$, \\ Jieun Song ${ }^{1}$, Junghyun Lee ${ }^{1}$, and Woonsup Shin ${ }^{1 *}$ \\ ${ }^{1}$ Department of Chemistry, Sogang University, Seoul 121-742, Korea \\ ${ }^{2}$ Department of Chemistry, School of Advanced Sciences, VIT University, Vellore-632014, India
}

\begin{abstract}
The development of non-precious metal based electrocatalysts is highly desired for the oxygen reduction reaction (ORR) as alternates to noble metal based ORR electrocatalysts. Herein, we report mononulcear copper(II) complex [CuLbpy]ClO 4 ( $\mathrm{L}=4$-[(2-hydroxyphenylimino)methyl]benzoic acid) containing poly(allylamine.HCl) polymer (PAlACuLbpy) as an electrocatalyst for oxygen reduction reaction (ORR). PAlACuLbpy was mixed with poly(acrylic acid) and tetraethylortho silicate to prepare a composite and then deposited on the screen printed electrode surface. The modified electrode (PAlACuLbpy/PCE) is highly stable and showed a quasi-reversible redox behavior with $\mathrm{E}_{1 / 2}=-0.2 \mathrm{~V}$ vs. $\mathrm{Ag} / \mathrm{AgCl}(3 \mathrm{M}$ $\mathrm{KCl}$ ) in $0.1 \mathrm{M}$ phosphate buffer at $\mathrm{pH} 7$ under argon atmosphere. PAlACuLbpy/PCE exhibited a remarkable ORR activity with an onset potential of $-0.1 \mathrm{~V}$ vs $\mathrm{Ag} / \mathrm{AgCl}$ in $0.1 \mathrm{M} \mathrm{PB}(\mathrm{pH} 7)$ in the presence of oxygen. The kinetics for ORR was studied by rotating disk voltammetry in neutral aqueous medium and the results indicated that the number of electrons involving in the ORR is four and the conversion products are water and hydrogen peroxide.
\end{abstract}

Keywords : Bio-mimicking, Hydrophilic polymer, Copper complex, Modified electrode, Oxygen reduction reaction

Received : 28 October 2016, Accepted : 12 December 2016

\section{Introduction}

Copper oxidase enzymes such as laccase, ceruloplasmin and ascorbateoxidase catalyze the fourelectron reduction of dioxygen to water coupled with substrate oxidation [1-2]. These multicopper oxidases contain three types of copper showing distinctly different EPR spectral features (types 1-3) with type 3 being a coupled binuclear copper. The type 2 and type 3 centers form a trinuclear copper cluster, which is the active site for both binding and multi-electron reduction of dioxygen to water [4-7]. The electrochemical oxygen reduction reaction is fundamentally important in the development of electrochemical devices such as fuel cell and zinc-air batteries and as electrochemical oxygen sensors [8-9].

*E-mail address: shinws@sogang.ac.kr, duraisarav@gmail.com DOI: https://doi.org/10.5229/JECST.2016.7.4.298
The copper containing enzyme laccase [10] and bilirubin oxidase [11] modified electrodes reduce oxygen directly to water at higher positive potentials. Heller et al., reported a fuel cell cathode based on laccase for the reduction of oxygen that operates with only $70 \mathrm{mV}$ of over potential [12]. Another class of efficient and the most promising electrocatalysts for oxygen reduction are the noble metal, platinum, platinum nano-particles and its alloys with other metals. The formation of $-\mathrm{OH}$ species which will hinder the ORR, the cost and relatively low abundance of $\mathrm{Pt}$ in nature are the drawbacks of the $\mathrm{Pt}$ based electrocatalysts [13-14].

The advancement of biomimetic chemistry attracts the researchers to utilize it for several applications like catalysts in organic transformations, electrocatalysts, etc., [15-19]. In this context, electrocatalytic reduction of oxygen is achieved by using metalloporphyrins, metallophthalocyanines and metal oxides and 
other non-precious metal electrocatalysts [20-21]. Recently, various efforts have been devoted to study the electrocatalytic activities of copper complexes [22] and copper complex derived electrode surfaces [23-25]. Several research groups have investigated the electrocatalytic performance of copper complexes with different ligands; (a) $\mathrm{Cu}$ (II) complex of 4,7-diphenyl1,10-phenanthroline disulfonate adsorbed on graphite electrodes [26-27] (b) $\mathrm{Cu}(\mathrm{II})$ hexaazamacrocyclic complex on glassy carbon electrode [28] (c) copper(II)2,4,6-tris(2-pyridyl)-1,3,5-triazine complex adsorbed on a graphite electrode [29] (d) copper(II)-tris(3aminopropyl)amine and imidazole complex on pyrolytic graphite electrode [30], (e) substituted-1,10phenonthroline-copper complexes on graphite [31] and edge-plane graphite electrodes [32]. The copper complex, 1,5-diaminonaphthalene copper was attached to the conducting polymer poly-terthiophene carboxylic acid, which further deposited on GCE and studied ORR [33]. Gewirth et al reported a very high ORR activity for a synthetic copper based electrocatalyst, copper complex of 3,5-diamino-1,2,4-triazole supported on carbon black [34].

In the present study, we intend to prepare a copper complex, which mimics the type 3 copper center by taking into account of biomimetic chemistry for oxygen reduction reaction. In the present study, we incorporated the redox-active mononuclear copper complex [CuLbpy $] \mathrm{ClO}_{4}(\mathrm{~L}=4-[(2-$ hydroxyphenylimino $)$ methyl $]$ benzoic acid) into hydrophilic poly(allylamine. $\mathrm{HCl}$ ) polymer backbone through amide linkage. The redox polymers derived from hydrophilic polymer have special characteristics such as when they are cross linked on the electrode, they become insoluble, but swell in water to form hydrogel. The increased segmental mobility and swelling upon hydration increase the frequency of electron-transferring collision between the redox centers and thus increase the electronic conductivity $[35,36]$. The precursors and polymer were characterized by FTIR and elemental analysis and cyclic voltammetric techniques. The electrochemical and electrocatalytic behavior towards ORR of the modified electrode were studied. The electrocatalytic kinetics of ORR was determined by rotating disc voltammetry.

\section{Experimental Section}

4-Formylbenzoicacid, 2-aminophenol, copper(II) perchlorate hexahydrate, poly(allylamine hydrochloride) (PAlA, Mw : 35,000), poly(acrylic acid) (PAA, Mw : 90,000), tetraethyl orthosilicate (TEOS), dimethylacetamide (DMAC) were purchased from Sigma-Aldrich and used without any further purification. (Caution: perchlorate salts are explosive; hence care should be taken while handling the compounds). Water purified from Milipore system was used in all experiments.

The synthetic procedures of the ligand and its mononuclear copper(II) complex, and their characterization are given in supplementary information.

\subsection{Synthesis of $[\mathrm{CuLbpy}] \mathrm{ClO}_{4}$ Incorporated PAIA Polymer (PAIACuLbpy)}

Poly(allylamine. $\mathrm{HCl})(0.1 \mathrm{~g})$ was dissolved in $10 \mathrm{~mL}$ deionzied water and $[\mathrm{CuLbpy}] \mathrm{ClO}_{4}(0.5$ mole equivalent to PAA. $\mathrm{HCl}$ monomer unit) in $10 \mathrm{~mL}$ methanol was added in the presence of EDC ( $0.4 \mathrm{~g}$; 0.5 mole equivalent). The resulting solution was stirred for $12 \mathrm{~h}$ and then, the resulting precipitate was filtered off and the solvent was removed under reduced pressure to get the product. The resulting copper incorporated polymer was washed several times with methanol to remove the un-reacted complex. The copper complex incorporated polymer PAlACuLbpy is soluble in hot water or dimethylacetamide.

\subsection{Synthesis of PAIACuLbpy-Poly(acrylic acid)- TEOS Composite}

The composite of PAlACuLbpy with polyacrylic acid and TEOS was prepared as follows. Solutions of PAlACuLbpy $(10 \mathrm{mg} / \mathrm{mL})$ and polyacrylic acid $(10 \mathrm{mg} / \mathrm{mL})$ were prepared by dissolving in DMAC and in water respectively. Then equal volume $(100 \mathrm{~mL})$ of the two solutions were mixed along with $40 \mathrm{~mL}$ of TEOS and stirred overnight to form the composite.

\subsection{Immobilization on PCE}

The immobilization of composite on plasma treated printed carbon electrode (PCE; SE100, Zensor R\&D, Taiwan, $\mathrm{id}=5 \mathrm{~mm}$, i.e., area $=0.196 \mathrm{~cm}^{2}$ ) was carried out by drop coating of $2 \mu \mathrm{L}$ of the polymercomposite. The electrode named as PAlACuLbpy/ PCE was dried at room temp and used for the further experiments.

\subsection{Spectroscopic Characterizations}

FT-IR spectra were recorded with Nicolet Avatar 
330FTIR (Thermo Electron Corporation) in dry $\mathrm{KBr}$. Electronic spectrum of $1 \%$ PAlACuLbpy in DMAC: water mixture(1:1) was recorded on a Agilent 8453 single beam spectrophotometer in the range $250-800 \mathrm{~nm}$. The Scanning Electron Microscopy (SEM) images were recorded with Hitachi S-4300 at $15 \mathrm{KV}$.

\subsection{Electrochemical Measurements}

Cyclic voltammetry (CV) experiments were performed with an electrochemical analyzer (CHI 900, $\mathrm{CH}$ Instrument Inc.) with three-electrode system comprising a glassy carbon $(3 \mathrm{~mm}$, Bioanalytical Systems) or PAlACuLbpy/PCE electrode $(5 \mathrm{~mm})$ as working electrode, $\mathrm{Ag} / \mathrm{AgCl}(3 \mathrm{M} \mathrm{KCl})$ as reference electrode and a platinum wire as counter electrode. Phosphate buffer $(0.1 \mathrm{M}, \mathrm{pH} 7)$ was used as an electrolyte solution. All measurements were done under argon, air and oxygen atmosphere at room temperature. In the case of the RDE and RRDE experiments, a glassy carbon rotating disk electrode was used as the working electrode and the disk area is $0.196 \mathrm{~cm}^{2}$. GC electrode was polished with $0.3 \mu \mathrm{m}$ alumina powder and cleaned by sonication in $50 \%$ ethanol solution before use.

\section{Results and Discussion}

The mononuclear copper(II) complex [CuLbpy $] \mathrm{ClO}_{4}$ is designed to mimic the activity of type 3 copper center to achieve the ORR . The synthesized copper complex was incorporated into polyallylamine polymer (structure 1) to immobilize on the electrode. In the first step, ligand and its mononuclear copper(II) complex containing free $\mathrm{COOH}$ group were synthesized (Supplementary data; scheme S1). In the next step, the mononuclear copper complex was condensed into polyallylamine through amide linkage and the polymer is having about $50 \%$ of complex modification. The polymer is well soluble in DMAC and hot water. The modification of poly(allylamine) by copper complex is confirmed by CHN analysis and FT-IR and UV-visible spectroscopy. From the elemental analysis, the percentage of $\mathrm{C}, \mathrm{H}$, and $\mathrm{N}$ was found to be $52.17,3.65$ and 9.7 respectively for PAlACuLbpy and these values are close to the extent of substitution of $50 \%$. The FT-IR spectrum of the polymer is shown in supplementary Fig. S2. The polymer showed the oximidato $\mathrm{C}=\mathrm{O}$ stretching

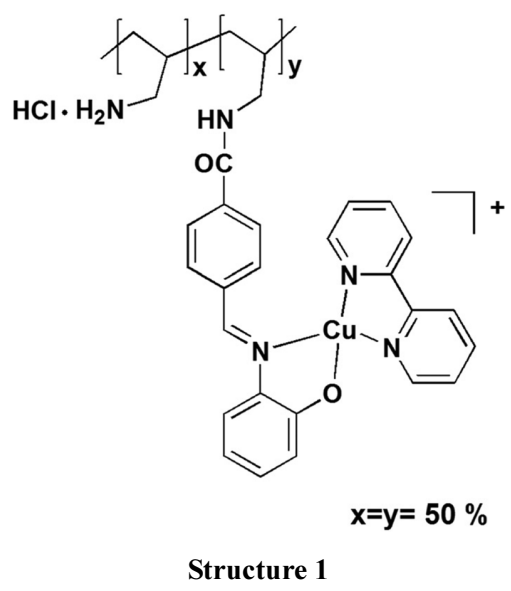

vibration at $1602 \mathrm{~cm}^{-1}$ along with $\mathrm{C}=\mathrm{N}$ stretching vibration $1686 \mathrm{~cm}^{-1}$, which prove the incorporation of copper complex. The polymer showed a single broad band at $1089 \mathrm{~cm}^{-1}$ ( $v_{3}$ antisymmetric stretching), which does not show any split, and a medium band at $650 \mathrm{~cm}^{-1}$ ( $v_{4}$ antisymmetric bending) due to uncoordinated perchlorate ion [37]. The UV-vis spectrum (Fig. S3 in supplementary) of polymer exhibited a d-d transition around $620 \mathrm{~nm}$ which is consistent with a tetracoordinate $\mathrm{Cu}$ atom in a nearly square-pyramidal geometry with weak coordination of water.

Polyelectrolyte composite of PAlACuLbpy was prepared with poly(acrylic acid) or poly(phosphonic acid) or poly(vinyl alcohol) in the ratio of 1:1 by mechanical mixing to fabricate the electrode. From these blending mixtures, a stable modification on PCE was not achieved as it is leaching out on continuous potential scan in phosphate buffer. A stable film on the electrode was obtained when PAlACuLbpy was mixed with poly(acrylic acid) and TEOS. TEOS forms a silica network on acid hydrolysis, consequently it entangles the PAlACuLbpy and poly(acrylic acid) tightly, lead to sol-gel as shown in scheme 1. Apart from silica network, the electrostatic interactions between the polymers PAlACuLbpy and poly(acrylic acid) viz $\mathrm{NH}_{2} \& \mathrm{COOH}$ groups further influences the stability of the electrode.

The physical appearance and surface characteristics of the sol-gel composite material were studied. Fig. 1 shows the SEM images of the composite material. As mentioned above, the composite with silica was prepared by the sol-gel method under acid catalysis. From the SEM images, it is clearly observed 


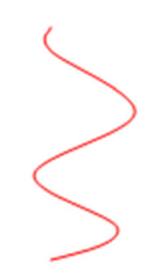

PAIA-CuLbpy

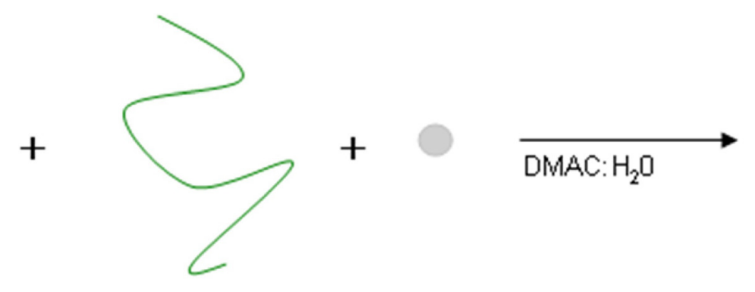

Polyacrylic acid

TEOS

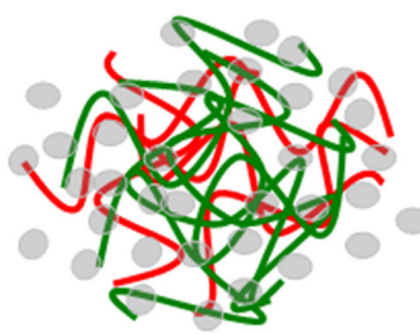

Scheme I
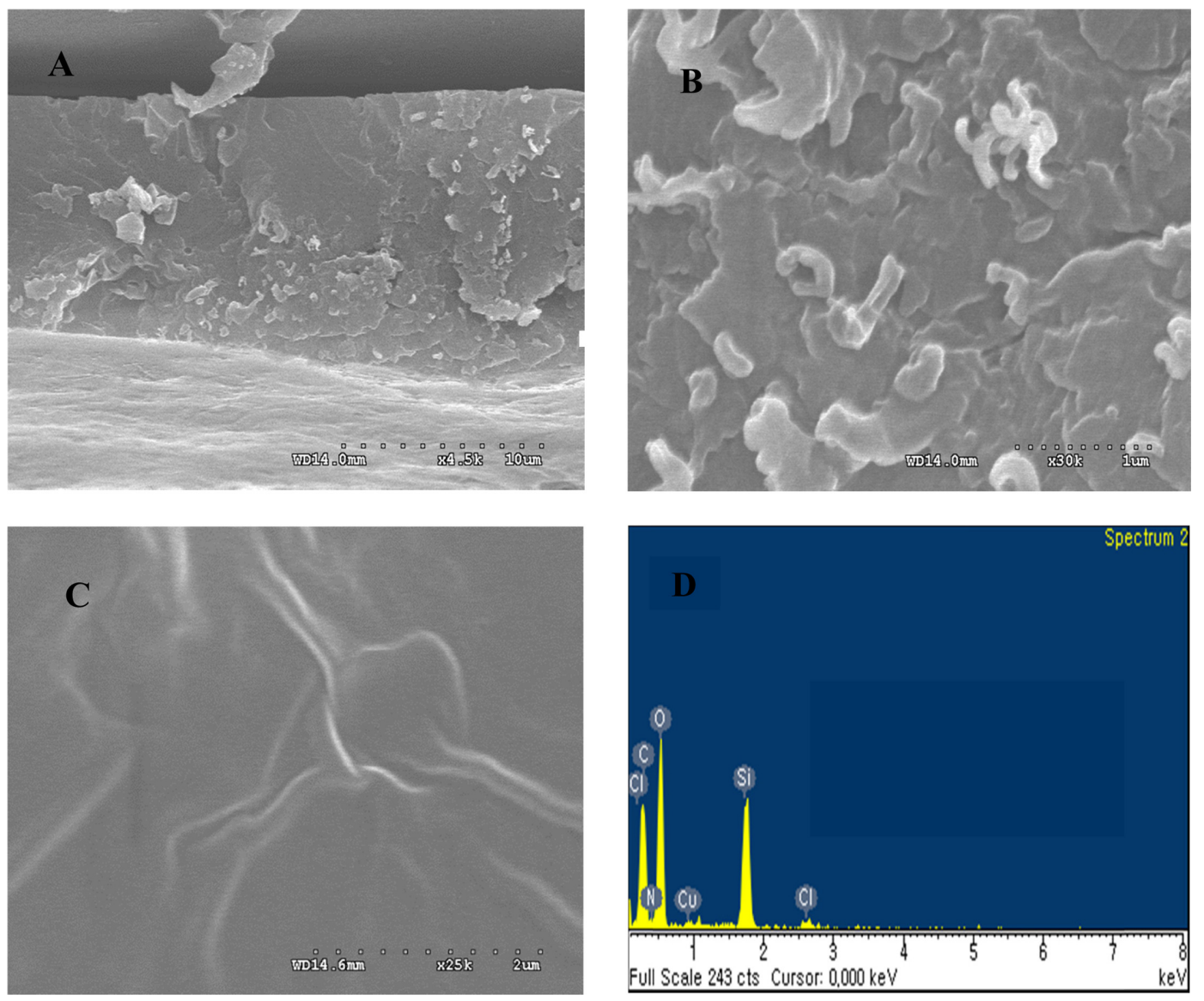

Fig. 1. SEM images of the PAlACuLbpy/PCE, cross-section (A\&B) and surface (C) EDAX (D) and Scale bars A: 20, B: 2, and $\mathrm{C}: 2 \mu \mathrm{m}$.

that the composite is crack free and homogeneous in nature. From the cross-section images, molecular level and even distribution of silica was noticed throughout the nanocomposite polymeric film. The sol-gel composite is free from any precipitation and any phase separation during it's preparation.
The cyclic voltammograms of PAlACuLbpycomposite coated on printed carbon electrode, PAlACuLbpy/PCE was recorded in $0.1 \mathrm{M}$ phosphate buffer at $\mathrm{pH} 7$ under argon atmosphere. The stability of the modified electrode was checked by carrying out the continuous cycling of 
voltammograms. A quasi-reversible redox couple with $\mathrm{E}_{1 / 2}=-0.2 \mathrm{~V}$ vs $\mathrm{Ag} / \mathrm{AgCl}(3 \mathrm{M} \mathrm{KCl})$ was observed which can be attributed to the redox couple of $\mathrm{Cu}(\mathrm{II}) / \mathrm{Cu}(\mathrm{I})$. The peak current of copper (II) reduction peak is not changed appreciably, which indicates that the modification of electrode is stable and not detachable from the electrode surface (supporting information S5). Fig. 2 shows the cyclic voltammograms of the modified electrode at different scan rates and presented in Fig. 3. The peak potential changed gradually when the scan rate is varied from $100 \mathrm{mV} / \mathrm{s}$ to $5 \mathrm{mV} / \mathrm{s}$. The cathodic peak was shifted to negative direction and anodic peak was shifted to more positive with increasing the scan rate. The plot of peak current versus scan rates is shown in the inset of Fig. 2 and the cathodic currents are proportional to the scan rate. This trend suggests that the electroactive copper complex is adsorbed on the electrode surface. The concentration of copper(II) species on the electrode film was calculated as follows; the charge consumed to reduce the film was estimated by integrating the anodic wave of $\mathrm{CV}$ at $2 \mathrm{mV} / \mathrm{s}$ and the amount of copper(II) species was calculated by Faraday's law assuming that the number of electron involved in the reduction process is 1 . The volume of the film was assumed to be $2 \mu \mathrm{L}$ which is the volume of the solution used to preparing the electrode by dropcoating. The concentration of the copper(II) species is found to be $7.8 \times 10^{-10}$ moles $/ \mathrm{cm}^{2}$.

\subsection{Electrocatalytic Oxygen Reduction Reaction}

It is obvious that copper containing enzymes and copper ion modified electrodes are showing electrocatalytic activity towards many biological processes [10-12] along with number of other biological species. Particularly, $\mathrm{Cu}$-containing fungal laccase enzymes reduce $\mathrm{O}_{2}$ directly to water at remarkable positive potentials. Herewith, the functional activity of $\mathrm{Cu}$-enzymes is mimicked by using the PAlACuLbpy/PCE in neutral aqueous medium. The modified electrode exhibited an excellent electrocatalytic activity towards oxygen reduction reaction (ORR) in phosphate buffer ( $\mathrm{pH} 7$ ) and observed a current corresponding to an electrocatalytic oxygen reduction under air and oxygen as shown in Fig. 3. It is known that the catalytic oxygen reduction peak appears along with copper(II) reduction peak with enhanced peak current as shown in Fig. 3 in the presence of air and oxygen. In the presence of oxygen, the catalytic current commenced at about $-0.1 \mathrm{~V}$, which is $0.3 \mathrm{~V}$ more positive than that of the bare PCE electrode (Fig. 3). When comparing the electrocatalytic currents under air with oxygen atmosphere, $\mathrm{i}_{\mathrm{O} 2} / \mathrm{i}_{\text {air }}$ is nearly 7 , showing a good accessibility of oxygen. Moreover, while removing the oxygen atmosphere by purging argon, the current of copper(II) peak of the modified electrode is returned back to its original position and this result indicates that the oxygen binds with the copper ion reversibly. The ORR takes place in the same region of similar reported copper complex-polymer modified electrodes, -0.05 to $0.2 \mathrm{~V}[33]$.

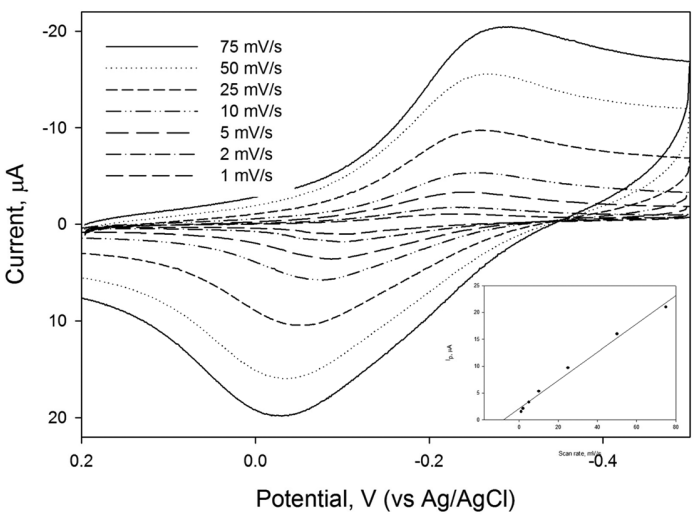

Fig. 2. Cyclic voltammograms of PAlACuLbpy/PCE at scan rates in $0.1 \mathrm{M}$ phosphate buffer, $\mathrm{pH} 7 \mathrm{vs} \mathrm{Ag} / \mathrm{AgCl}(3 \mathrm{M} \mathrm{KCl})$ under argon atmosphere; inset: peak current vs scan rates.

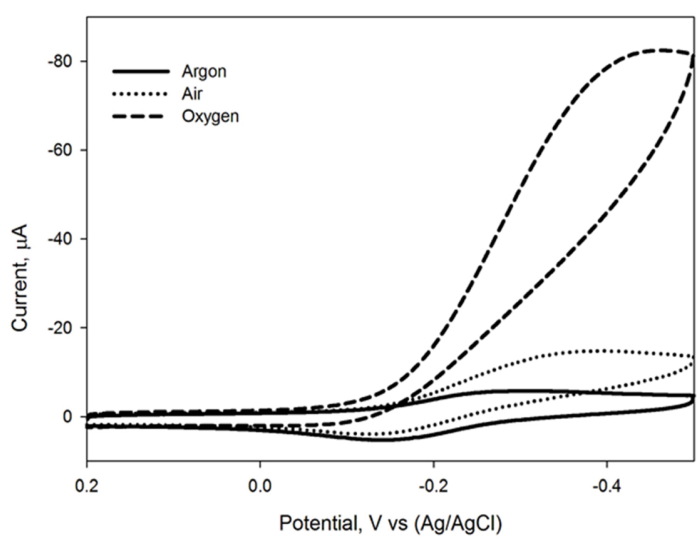

Fig. 3. Cyclic voltammograms of oxygen reduction on PAlACuLbpy/PCE in $0.1 \mathrm{M}$ phosphate buffer, $\mathrm{pH} 7$ at $5 \mathrm{mV} / \mathrm{s}$ vs $\mathrm{Ag} / \mathrm{AgCl}(3 \mathrm{M} \mathrm{KCl})$ under oxygen and air. 


\subsection{Hydrodynamic studies}

A kinetic study of the electrocatalytic oxygen reduction was performed with rotating disc (RDE) electrode modified with PAlACuLbpy-composite. Fig. 4 shows RDE voltammograms of PAlACuLbpy/ PCE in $0.1 \mathrm{M}$ phosphate buffer ( $\mathrm{pH} 7)$ saturated with oxygen at various rotating rates from 0 to $1500 \mathrm{rpm}$ with an increment of $300 \mathrm{rpm}$. The catalytic currents responsible for oxygen reduction start to flow around $-0.1 \mathrm{~V}$. A single stage of limiting current is observed when the potential is scanned from 0 to $-0.6 \mathrm{~V}$, indicating the electrocatalytic reduction of oxygen on PAlACubpy/PCE is controlled by the diffusion process. The Koutecky-Levich plots obtained from the RDE voltammograms are shown in Fig. 5, which are calculated at $-0.5 \mathrm{~V}$.

The diffusion limited currents for $\mathrm{O}_{2}$ reduction at the rotating disk electrode were calculated by the Levich equation employing the following parameters: diffusion coefficient of $\mathrm{O}_{2}, D_{\mathrm{O} 2}=2.5 \times 10^{-5} \mathrm{~cm}^{2} \mathrm{~s}^{-}$ ${ }^{1}$, kinematic viscosity of aqueous solution, $\mathrm{n}=0.01$ $\mathrm{cm}^{2} \mathrm{~s}^{-1}$, concentration of oxygen in oxygen-saturated solution, $C_{\mathrm{O} 2}=2.9 \times 10^{-4} \mathrm{~mol} \mathrm{~cm}^{-3}$, the Faraday constant, $F=96,485 \mathrm{C} / \mathrm{mol}$, and area of the disk electrode, $A=0.186 \mathrm{~cm}^{2}$

$$
i_{\lim }=0.620 n F A D^{2 / 3} \omega^{1 / 2} v^{-1 / 6} C_{O_{2}}
$$

From the slope of the Koutechy-Levich plot ($0.5 \mathrm{~V})$, the number of electrons involved in the catalytic reduction of $\mathrm{O}_{2}$ is calculated to be 3.8, which indicates that the catalytic reduction of oxygen on PAlACulbpy/PCE is four electron process. The electroreduction of oxgen on a bare GCE is known to be around 2 electron process [37]. The extrapolated Koutechy-Levich plot is not passing through zero, which is probably due to the equilibrium between $\mathrm{O}_{2}$ and $\mathrm{H}_{2} \mathrm{O}_{2}$. Both two and four electron reduction of oxygen is previously reported for $\mathrm{Cu}$ (II)-complex adsorbed EPG surfaces [26,38]. From the kinetic studies, it is observed that the electroreduction of $\mathrm{O}_{2}$ was catalyzed by surface adsorbed $\mathrm{Cu}$ complex, through an unstable dioxygen adduct as an intermediate, proceeded by an inner sphere mechanism. Based on the above results, the following inner-sphere mechanism is proposed for the electrocatalytic reduction of $\mathrm{O}_{2}$.

$\mathrm{PAlACu}(\mathrm{II}) \mathrm{Lbpy} / \mathrm{PCE}+\mathrm{e}^{-} \leftrightarrow \mathrm{PAlACu}(\mathrm{I}) \mathrm{Lbpy} / \mathrm{PCE}^{-}$

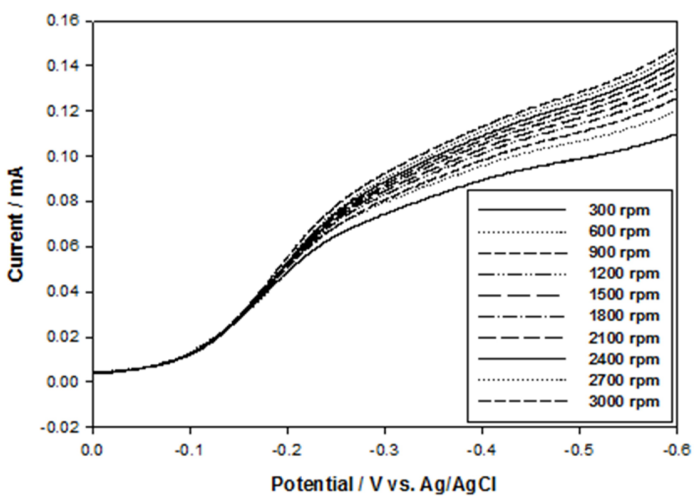

Fig. 4. Current-potential curves for the oxygen reduction on a PAlACuLbpy/RDE in $0.1 \mathrm{M}$ phosphate buffer, $\mathrm{pH} 7$ at $5 \mathrm{mV} / \mathrm{s}$ under oxygen.

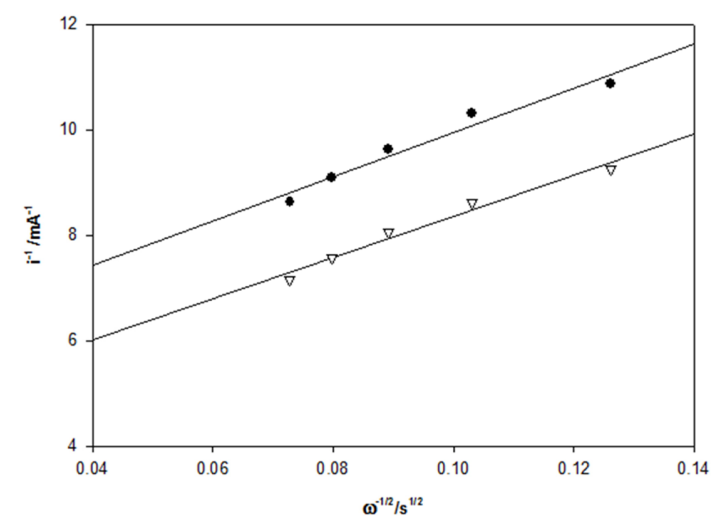

Fig. 5. Koutecky-Levich plot for the oxygen reduction with a PAlACuLbpy/RDE (at $-0.5 \mathrm{~V}$ ) in $0.1 \mathrm{M}$ phosphate buffer, $\mathrm{pH} 7$ at $5 \mathrm{mV} / \mathrm{s}$. (O) Two electron and $(\nabla)$ four electron process.

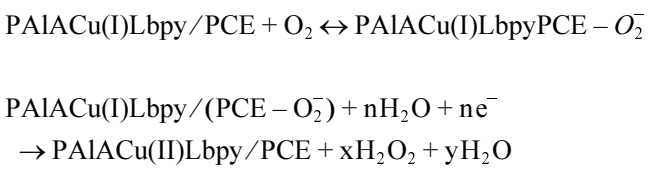

A sensitive assessment was carried out to obtain the reaction stoichiometry by a rotating ring disc electrode RRDE. The potential of the ring was kept constant at $1.0 \mathrm{~V}$ to detect the generation of $\mathrm{H}_{2} \mathrm{O}_{2}$. The upper part of Fig. 6 shows the current-potential curve for the reduction of $\mathrm{O}_{2}$ at PAlACuLbpy modified disk electrode. In the lower part of 6 , the corresponding anodic current at the Pt ring electrode indicates that the reduction of $\mathrm{O}_{2}$ at the disk produces 


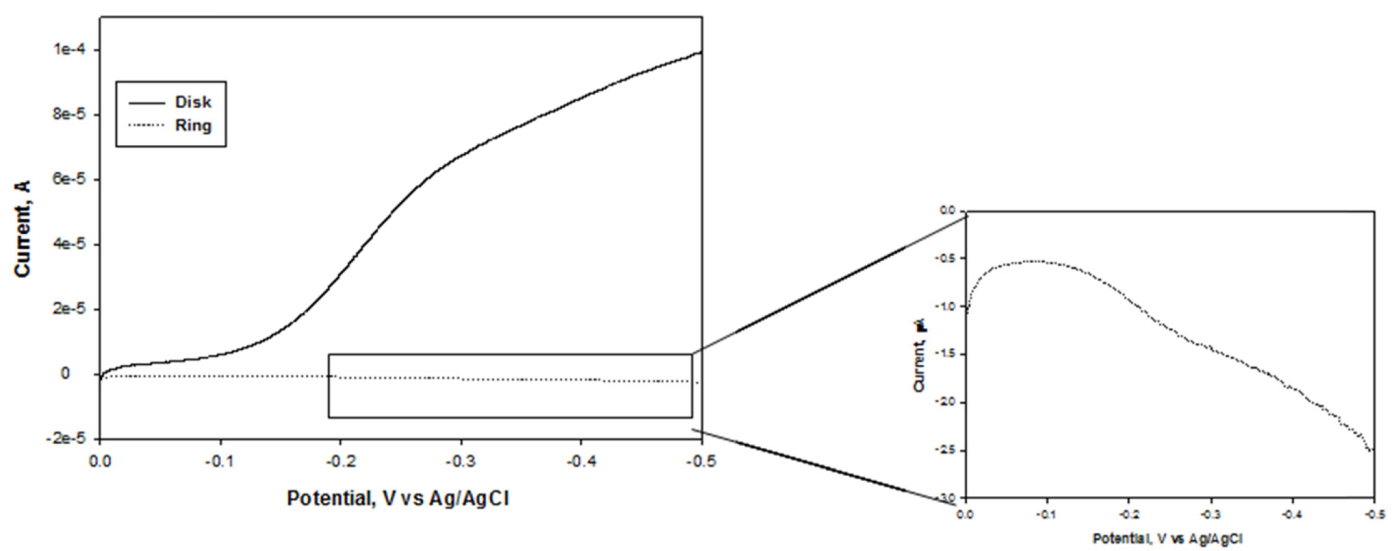

Fig. 6. Current-potential curves for the oxygen reduction on a $\mathrm{RDE}(0.1 \mathrm{M}$ phosphate buffer, $\mathrm{pH} 7,50 \mathrm{mV} / \mathrm{s})$.

both $\mathrm{H}_{2} \mathrm{O}$ and $\mathrm{H}_{2} \mathrm{O}_{2}$. This observation suggests the possibility of a parallel catalytic pathway where oxygen is reduced to $\mathrm{H}_{2} \mathrm{O}$ and $\mathrm{H}_{2} \mathrm{O}_{2}$.

\section{Conclusion}

A hydrophilic mononuclear copper(II) complex containing poly(allylamine. $\mathrm{HCl})$ polymer was synthesized. Composite of polymer with poly(acrylic acid) and TEOS was prepared to modify the electrode surface. A stable modification on PCE was obtained by drop coating the composite. The modified electrode PAlACuLbpy/PCE exhibited a quasireversible redox couple with $\mathrm{E}_{1 / 2}=-0.2 \mathrm{~V}$ vs. $\mathrm{Ag} /$ $\mathrm{AgCl}(3 \mathrm{M} \mathrm{KCl})$ in $0.1 \mathrm{M}$ phosphate buffer ( $\mathrm{pH} 7)$. The modified electrode PAlACuLbpy/PCE is utilized for ORR and it showed an excellent electrocatalytic response for the reduction of oxygen in phosphate buffer ( $\mathrm{pH} 7)$ under oxygen at onset of $0.1 \mathrm{~V}$, which is $0.3 \mathrm{~V}$ more positive than bare electrode. The kinetics involved in the catalytic reduction of oxygen was studied by rotating disk voltammetric technique. The results indicated that the number of electrons involved in the ORR are four with conversion of water and $\mathrm{H}_{2} \mathrm{O}_{2}$.

\section{Acknowledgment}

This work was supported by the Korea Institute of Energy Technology Evaluation and Planning (KETEP) and the Ministry of Trade, Industry \& Energy (MOTIE) of the Republic of Korea (No. 20142010201800). D.S. acknowledges the Science and Engineering Research
Board (SERB), Government of India (SB/FT/CS-168/ 2013) for the financial assistance.

\section{References}

[1] E. I. Solomon, U. M. Sundaram, T. E. Machonkin, Chem. Rev., 1996, 96(7), 2563-2606.

[2] L. Que Jr., Y. Watanabe, Science 2001, 292(5517), 651653.

[3] E. I. Solomon, P. Chen, M. Metz, S.K. Lee, A. E. Palmer, Angew. Chem. Int. Ed., 2001, 40(24), 45704590.

[4] R. H. Holm, P. Kennepohl, E. I. Solomon, Chem. Rev., 1996, 96(7), 2239-2314.

[5] I. E. Marko, M. Tsukazaki, P. R. Giles, S. M. Brown, C. J. Urch, Angew. Chem. Int. Ed., 1997, 36(20), 22082210.

[6] E. I. Solomon, P. M. Jones, J. A. May, Chem. Rev., 1993, 93(8), 2623-2644.

[7] E. I. Solomon, F. Tuczek, D. E. Root, C. A. Brown, Chem. Rev., 1994, 94(3), 827-856.

[8] S.B. Adler, Chem. Rev., 2004, 104(10), 4791-4844.

[9] J.W. Shie, U. Yogeswaran, S. Chen, Talanta 2009, 78(3), 896-902.

[10] N. Mano, V. Soukharev, A. Heller, J. Phys. Chem. B, 2006, 110(23), 11180-11187.

[11] G. Merle, A. Habrioux, K. Servat, M. Rolland, C. Innocent, K.B. Kokoh, S. Tingry, Electrochim. Acta 2009, 54(11), 2998-3003.

[12] V. Soukharev, N. Mano, A. Heller, J. Am. Chem. Soc., 2004, 126(27), 8368-8369.

[13] C.W.B. Bezerra, L. Zhang, H. Liu, K. Lee, A.L.B. Marques, E. P. Marques, H. Wang, J. Zhang, J. Power Sources 2007, 173, 891-908.

[14] M. A. Rigsby, W.P. Zhou, A. Lewera, H.T. Duong, P.S. Bagus, W. Jaegermann, R. Hunger, A. Wieckowski, J. Phys. Chem. C, 2008, 112(39), 15595-15601. 
[15] M. Broring, Angew. Chem. Int. Ed., 2007, 46(33), 62226224.

[16] Z. Varpness, J. W. Peters, M. Young, T. Douglas Nano Lett., 2005, 5(11), 2306-2309.

[17] R. Reslow, Acc. Chem. Res., 1995, 28(3), 146-153.

[18] T. Punniyamurthy, S. Velusamy, J. Iqbal Chem. Rev., 2005, 105(6), 2329-2364.

[19] V. Artero, M. Fontecave, Coord. Chem. Rev., 2005, 249(15), 1518-1535.

[20] Y. Feng, N. Alonso-Vante, Phys. Stat. Sol. (b), 2008, 245(9), 1792-1806.

[21] R. Bashyam, P. Zelenay, Nature 2006, 443(7107), 63-66.

[22] M.A. Thorseth, C.E. Tornow, E.C.M. Tse, A.A. Gerwith, Coord. Chem. Rev., 2013, 257(1), 130-139.

[23] F.F. Wang, P.J. Wei, G.Q. Yu, J.G. Liu, Chem. Eur. J., 2016, 22(1), 382-389.

[24] R. C. Wang, T.L. Yin, P.J. Wei, J.G. Liu, RSC $A d v$., 2015, 5(81), 66487-66493.

[25] Y.T. Xi, P.J. Wei, R.C. Wang, J.G. Liu, Chem. Commun., 2015, 51(35), 7455-7458.

[26] J. Zhang, F.C. Anson, J. Electroanal. Chem., 1993, 348(1), 81-97.

[27] J. Zhang and F.C. Anson, J. Electroanal. Chem., 1992, 331(1-2), 945-957.

[28] K. Slowinski, Z. Kublik, R. Bilewicz, M.
Pietraszkiewicz J. Chem. Soc., Chem. Commun., 1994, 9, 1087-1088.

[29] V.L.N. Dias, E.N. Fernandes, L.M.S. da Silva, E. P. Marques, J. Zhang, A.L. B. Marques, J. Power Sources 2005, 142(1), 10-17.

[30] C. X. Cai, K. H. Xue, X. Y. Xu, Q. H. Luo, J. Appl. Electrochem., 1997, 27(7), 793-798.

[31] Y. Lei, F. C. Anson, Inorg. Chem., 1995, 34(5), 10831089.

[32] C. C. L. McCrory, X. Ottenwaelder, T. D. P. Stack, C. E. D. Chidsey, J. Phys. Chem. A, 2007, 111(49), 1264112650.

[33] M. Boopathi, M. S. Won, Y. H. Kim, S. C. Shin, Y.B. Shim, J. Electrochem. Soc., 2002, 149(7), E265-E271.

[34] M.S. Thorum, J. Yadav, A.A. Gewirth, Angew. Chem. Int. Ed., 2009, 121, 171.

[35] A. Heller, Curr. Opin. Chem. Biology, 2006, 10(6), 664672.

[36] J. Ribas, C. Diaz, R. Costa, J. Tercero, X. Solans, M. Font-Bardia, H. Stoeckli-Evans, Inorg. Chem., 1998, 3, 7233.

[37] K. Vaik, D.J. Schiffrin, K. Tammeveski, Electrochem. Commun. 2004, 6(1), 1-5.

[38] J.J. Zhang, F.C. Anson, Electrochim. Acta 1993, 38(16), 2423-2429. 